\title{
Avascular necrosis of the femoral head in patients receiving intermittent cytotoxic and corticosteroid therapy for Hodgkin's disease
}

\author{
B. W. HANCOCK \\ M.D., D.C.H., M.R.C.P.

\begin{abstract}
Summary
Two patients with stage IIIB Hodgkin's disease are reported, in whom treatment with cytotoxic and corticosteroid therapy was followed by avascular necrosis of the femoral head.
\end{abstract}

\author{
P. HUCK* \\ M.C.R., F.R.C.R.
}

\author{
B. Ross $\dagger$ \\ M.D., F.R.C.R., D.M.R.D. \\ University Department of Medicine, Royal Hospital, Sheffield; *Division of Radiotherapy and \\ $\dagger$ Department of Radiology, Weston Park Hospital, Sheffield
}

\section{Introduction}

Chemotherapy in patients with generalized malignant lymphoma involves the use of cyclical regimes of drug combinations many of which incorporate intermittent corticosteroid therapy. There have been two reports (Ihde and DeVita, 1975; Sweet et al., 1976) in the American literature of avascular necrosis of the femoral head occurring during or after such therapy and incriminating corticosteroids as the cause. Two further cases of a similar nature are now reported.

\section{Case reports}

Case 1

A man aged 32 years presented in July 1973 with generalized lymphadenopathy diagnosed as mixed cellularity Hodgkin's disease (Stage IIIB). He had six courses of intensive MOPP quadruple cytotoxic chemotherapy (intravenous mustine $6 \mathrm{mg} / \mathrm{m}^{2}$ and vincristine (Oncovin) $1.4 \mathrm{mg} / \mathrm{m}^{2}$ on days 1 and 8 ; oral procarbazine $100 \mathrm{mg} / \mathrm{m}^{2}$ and prednisolone 40 $\mathrm{mg} /$ day for 14 days; 28 days between each course). Complete remission of Hodgkin's disease was seen but 3 months after cessation of intensive chemo- therapy the patient complained of pain in the left hip. Radiographs (including tomograms) revealed avascular necrosis of the femoral head. Successful hip replacement was performed in October, 1975, and at the present time (December, 1977) the patient remains completely well.

\section{Case 2}

A 15-year-old girl presented in January 1975 with Stage IIIB Hodgkin's disease (nodular sclerosing type) in association with digital clubbing and hypertrophic pulmonary osteo-arthropathy (HPOA) (Hancock et al., 1976). She was treated with six courses of combination chemotherapy (initially MOPP, as in case 1; later vinblastine (Velbe) was substituted for vincristine since peripheral neurotoxicity was observed). Following intensive chemotherapy the HPOA findings resolved but residual mediastinal and inguinal lymphadenopathy were noted and the patient received total nodal irradiation followed by three further monthly cycles of MVPP with good response. During the third of these courses the patient complained of pain in the right hip and $\mathrm{X}$-ray of the pelvis at this stage was normal (Fig. 1a). Five months later she again complained of pain; on this occasion radiographs (and tomograms) showed avascular necrosis of the right femoral head (Fib. 1b). This patient has recently (1978) died after severe relapse of disseminated Hodgkin's disease; post-mortem confirmed the presence of avascular necrosis. 


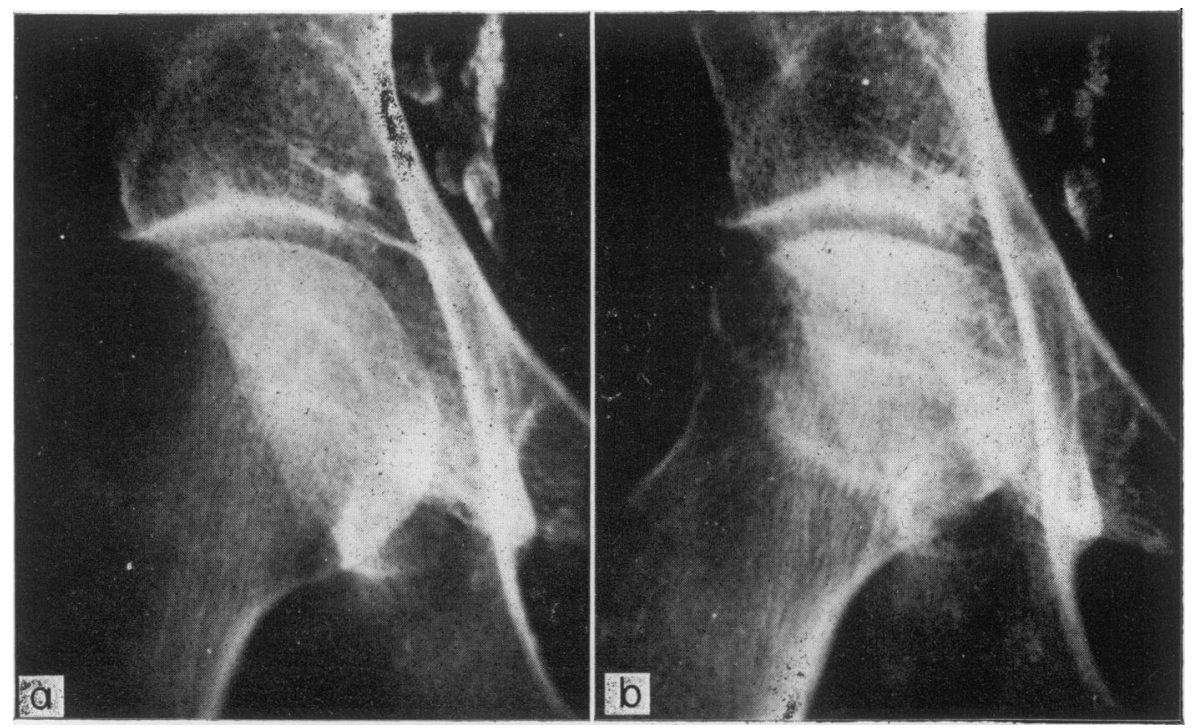

FIG. 1. Radiographs of right hip joint in case 2 showing (a) normal joint at onset of hip pain; (b) avascular necrosis of the femoral head 5 months later.

\section{Discussion}

It is possible that intermittent corticosteroid therapy was responsible for the avascular necrosis in these patients even though both had received other cytotoxic drugs and although the second patient had previously exhibited another bone disorder (HPOA) and did receive standard total nodal irradiation.

Avascular necrosis of the femoral head is a recognized complication of steroid therapy; in general, patients subjected to high dose or prolonged therapy appear to be at greater risk than those receiving small doses but the complication may be seen when total steroid dose, daily dose, and duration of treatment are minimal (Sutton, 1968). It is perhaps not surprising, therefore, that intermittent corticosteroid therapy (as part of combination cytotoxic chemotherapy) should occasionally be associated with avascular necrosis. Hip pain in such patients should not necessarily be attributed to bone involvement from lymphoma, and expert radiological advice should be sought.

\section{Acknowledgments}

We are grateful to Dr I. G. Emmanuel and Professor J. Richmond for allowing us to report details of case 2. B.W.H. is financially supported by the Cancer Research Campaign (Yorkshire Branch).

\section{References}

Hancock, B.W., Richmond, J., Powell, T. \& Emmanuel, I.G. (1976) Intra-thoracic Hodgkin's disease presenting as hypertrophic osteoarthropathy. British Journal of Radi$o \log y, 49,647$.

IHDE, D.C. \& DeVITA, V.T. (1975) Osteonecrosis of the femoral head in patients with lymphoma treated with intermittent combination chemotherapy (including corticosteroids). Cancer, 36, 1585.

Sutton, R.D. (1968) Aseptic necrosis of the bone, a complication of corticosteroid therapy. In: Drug-Induced Diseases, Vol. 3, (Ed. by Meyler, L. \& Peck, H.M.) pp. 171-176. Excerpta Medica Foundation, Amsterdam.

Sweet, D.L., Roth, D.G., Desser, R.K. \& Ultmann, J.E. (1976) Avascular necrosis of the femoral head during combination chemotherapy. Annals of Internal Medicine, 85, 67. 